\title{
Kinetics of chiral transitions and domain growth in quark matter
}

\author{
Awaneesh Singh, Sanjay Puri \\ School of Physical Sciences, Jawaharlal Nehru University, \\ New Delhi-110067, India.

\section{Hiranmaya Mishra*} \\ Theory Division, Physical Research Laboratory, Navrangpura, Ahmedabad - 380009, India. \\ E-Mail: hmeprl.res.in
}

We study the kinetics of chiral transitions in quark matter within the framework of Nambu-JonaLasinio model.We do a Ginzburg Landau expansion of NJL model thermodynamic potential. The same is used in a Langevin description for the order parameter evolution for chiral transition. The Langevin equation includes both dissipative as well as inertial terms. We focus on the effect of inertial terms on the coarsening dynamics subsequent to a quench from the massless quark phase to the massive quark phase. The domain growth process shows a crossover from a fast inertial regime [with $L(t) \sim t(\ln t)^{1 / 2}$ ] to a diffusive Cahn-Allen regime [with $L(t) \sim t^{1 / 2}$ ].

\footnotetext{
*Speaker.
} 
Heavy-ion collision experiments at high energies produce hot and dense strongly-interacting matter, and provide an opportunity to explore the phase diagram of quantum chromodynamics (QCD) in the plane of temperature $(T)$ and baryon chemical potential $(\mu)$. Many model studies [1], as well as recent lattice studies [2], indicate that at sufficiently large baryonic densities, there is a line of first-order transitions in the $(\mu, T)$-plane between a chirally-symmetric phase and a brokensymmetry phase. As one moves along the phase boundary towards higher $T$ and smaller $\mu$, the first-order transition becomes weaker - ending in a tricritical point (TCP) in the limit of vanishing current quark mass or a rapid crossover for non-zero current quark mass [3]. For even smaller values of $\mu$, there is a line of second-order transitions. While the high- $T$ and small- $\mu$ region of the QCD phase diagram has been explored in recent experiments, future heavy-ion collision experiments plan to explore the high baryon density regime, particularly the region around the TCP [4].

It is important to stress here that heavy-ion experiments are essentially nonequilibrium processes. It is challenging to extract the thermodynamic properties of quark-hadron phase transition from nuclear collision experiments due to the absence of global thermal equllibrium. This is because nonequllibrium effects play an important role in the evolution of the fire ball. Therefore, an understanding of the equilibrium phase diagram alone is not sufficient to discuss the properties of the system. One also has to understand the kinetic processes which drive the phase transition, and the properties of the nonequilibrium structures that the system goes through to reach equilibrium. In this context, both the critical dynamics and the far-from-equilibrium kinetics, of the chiral transition have attracted much recent attention. In the study of critical dynamics (i.e. the temporal behaviour in the vicinity of critical point), much interest has been focussed upon the signatures of the critical end point(CEP) of QCD. In the present work, on the otherhand, we focus on the far from equllibrium kinetics, i.e. the evolution of the system after a quench from a disordered phase to an ordered phase with non-vansihing quark-anti-quark condensates. In this context, the relaxation to equilibrium using Langevin equation has been attempted in Ref. [5], where the authors studied the early time dynamics of the spinodal decomposition and the effect of dissipation on the spinodal instability. Further, the bubble nucleation kinetics in chiral transition was studied by Bessa etal [6]. A time-dependent Ginzburg Landau equation (TDGL) was derived in Ref. [7] starting from a non-ideal, nonrelativistic hydrodynamics for coupled order parameters. The authors here clarified the effect of viscosity in the ordering kinetics. Further, Randrup [8], studied the amplification of the spinodal fluctuation within a fluid dynamical model for the nuclear collisions. Here, the study was mostly focused on the evolution in the linearized regime which showed an exponential growth of the initial fluctuations.

Recently, we initiated a study of far-from equillibrium kinetics of chiral phase transition $[9,10]$. Our approach has been are complementary to Refs. $[5,6,7,8]$. Our study investigates the late stages of phase-separation kinetics in quark matter and the scaling properties of emergent morphologies. The system is described by nonlinear evolution equations in this regime: the exponential growth of initial fluctuations is saturated by the nonlinearity.

To model chiral symmetry breaking in QCD, we use the two-flavor Nambu-Jona-Lasinio (NJL) 
model $[11,12]$. The thermodynamic potential in terms of the "constituent" mass $\mathrm{M}$ is given as[9]

$$
\begin{aligned}
\tilde{\Omega}(M, \beta, \mu)=-\frac{12}{(2 \pi)^{3} \beta} \int d \vec{k} & \left\{\ln \left[1+e^{-\beta\left(\sqrt{k^{2}+M^{2}}-\mu\right)}\right]\right. \\
& \left.+\ln \left[1+e^{-\beta\left(\sqrt{k^{2}+M^{2}}+\mu\right)}\right]\right\} \\
& -\frac{12}{(2 \pi)^{3}} \int d \vec{k}\left(\sqrt{k^{2}+M^{2}}-k\right)+\frac{M^{2}}{4 G}
\end{aligned}
$$

where $\beta=\left(k_{B} T\right)^{-1}$. Here, we have taken vanishing current quark mass $(m=0)$, and introduce $M=-2 G \rho_{s}$, with $\rho_{s}=\langle\bar{\psi} \psi\rangle$ being the scalar density . The NJL model parameters the four ferion coupling $G$ and the three momentum cutoff $\Lambda$ are fixed such that the pion decay constant and the phenomenological values for the quark condensates are reproduced. In the present work, we have set the three momentum ultra violet cut-off $\Lambda=653.3 \mathrm{MeV}$ and the four fermion coupling $G=5.02 \times 10^{-6} \mathrm{MeV}^{-2}$. With these values the constituent quark mass turns out to be $M=312$ $\mathrm{MeV}$. With these parameters chiral symmetry is restored at $T \simeq 190 \mathrm{MeV}$ at zero density with a second order phase transition. Similarly a first order transition takes place at zero temperature for quark chemical potential $\mu \simeq 326.3 \mathrm{MeV}$. In the $T-\mu$ plane, the tricritical point lies at $\left(\mu_{t c p}, T_{t c p}\right)=$ $(282.6,78) \mathrm{MeV}$ where the first order line meets the second order transition line.

The potential in Eq. (1) may be expanded as a Landau potential in the order parameter $M$ :

$$
\tilde{\Omega}(M)=\tilde{\Omega}(0)+\frac{a}{2} M^{2}+\frac{b}{4} M^{4}+\frac{d}{6} M^{6}+O\left(M^{8}\right) \equiv f(M),
$$

correct up to logarithmic factors [12]. In the following, we consider the expansion of $\tilde{\Omega}(M)$ up to the $M^{6}$-term. This will be adequate to capture the salient features of the NJL model phase diagram, as we see shortly. The first two coefficients in Eq. (2) can be obtained by comparison with Eq. (1) as

$$
\begin{gathered}
\tilde{\Omega}(0)=-\frac{6}{\pi^{2} \beta} \int_{0}^{\Lambda} d k k^{2}\left\{\ln \left[1+e^{-\beta(k-\mu)}\right]+\ln \left[1+e^{-\beta(k+\mu)}\right]\right\}, \\
a=\frac{1}{2 G}-\frac{3 \Lambda^{2}}{\pi^{2}}+\frac{6}{\pi^{2}} \int_{0}^{\Lambda} d k k\left[\frac{1}{1+e^{\beta(k-\mu)}}+\frac{1}{1+e^{\beta(k+\mu)}}\right] .
\end{gathered}
$$

We treat the higher coefficients as phenomenological parameters, which are obtained by fitting $\tilde{\Omega}(M)$ in Eq. (2) to the integral expression for $\tilde{\Omega}(M)$ in Eq. (1) [9]. There are two free parameters in the microscopic theory ( $\mu$ and $T$ ), so we consider the $M^{6}$-Landau potential with parameters $b$ and $d$. For stability, we require $d>0$.

The extrema of the potential in Eq. (2) are determined by the gap equation: $f^{\prime}(M)=a M+$ $b M^{3}+d M^{5}=0$. The corresponding solutions are $M=0$, and $M_{ \pm}^{2}=\left(-b \pm \sqrt{b^{2}-4 a d}\right) /(2 d)$. The phase diagram for the Landau potential is shown in Fig. 1. (A) For $b>0$, the transition is secondorder, analogous to an $M^{4}$-potential - the stationary points are $M=0$ (for $a>0$ ) or $M=0, \pm M_{+}$ (for $a<0$ ). For $a<0$, the preferred equilibrium state is the one with massive quarks. (B) For $b<0$, the solutions of the gap equation are as follows: (i) $M=0$ for $a>|b|^{2} /(4 d)$, (ii) $M=0$, $\pm M_{+}, \pm M_{-}$for $|b|^{2} /(4 d)>a>0$, and (iii) $M=0, \pm M_{+}$for $a<0$. A first-order transition takes place at $a_{c}=3|b|^{2} /(16 d)$ with the order parameter jumping discontinuously from $M=0$ to 


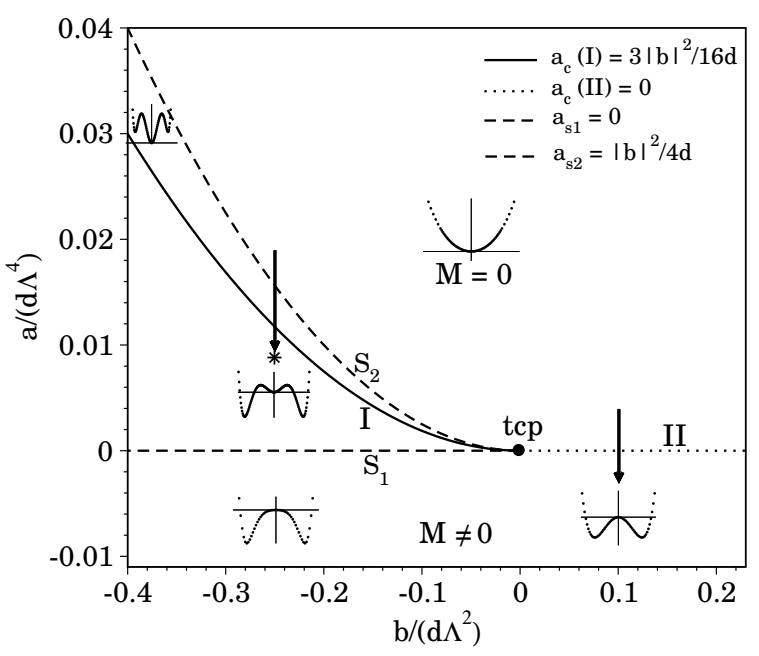

Figure 1: Phase diagram for the Landau free energy in in the $\left[b /\left(d \Lambda^{2}\right), a /\left(d \Lambda^{4}\right)\right]$-plane. A line of first-order transitions (I) meets a line of second-order transitions (II) at the tricritical point (tcp), which is located at $a=b=0$. The equations for I and II are specified in the figure. The dashed lines denote the spinodals $S_{1}$ and $S_{2}$, whose equations are also provided. The typical forms of the Landau potential in various regions are shown in the figure. The asterisk denotes the point where we quench the system for $b<0$ (first-order quench). The second-order quench studied here corresponds to $b /\left(d \Lambda^{2}\right)=1.269, a /\left(d \Lambda^{4}\right)=-0.225$, and is not shown in the figure for clarity.

$M= \pm M_{+}= \pm(3|b| / 4 d)^{1 / 2}$. The tricritical point is located at $b_{\mathrm{tcp}}=0, a_{\mathrm{tcp}}=0$. The dotted lines in Fig 1 denote the spinodals $S_{1}$ and $S_{2}$, with equations $a_{S_{1}}=0$ and $a_{S_{2}}=|b|^{2} /(4 d)$.

Next, let us study dynamical problems in the context of the above a heavy-ion collision. If the evolution is slow compared to the typical equilibration time, the order parameter field will be in local equilibrium. On the otherhand, if the expansion is fast enough, the field configuration will not be in thermal equllibrium and will lag in the sense that it will find itself in a more disordered state than the equllibrium configuration. We consider a system which is rendered thermodynamically unstable by a rapid quench from the massless phase to the massive phase in Fig. 1. The unstable massless state evolves via the emergence and growth of domains rich in the preferred massive phase $[14,15]$. Ther has been extensive study on such far from equllibrium dynamics and domain growth processs in the areas of condensed matter systems like magnets, alloys, fluids, liquid crystals as well as superconductors. However, equally fascinating problems are associated with kinetics of phase transitions associated in high energy physics and cosmology [16].

The coarsening system is inhomogeneous, and we account for this by including a surface tension term in the Landau free energy:

$$
\Omega[M]=\int d \vec{r}\left[\frac{a}{2} M^{2}+\frac{b}{4} M^{4}+\frac{d}{6} M^{6}+\frac{K}{2}(\vec{\nabla} M)^{2}\right] .
$$

It is customary to model the kinetics by the TDGL equation, which models the overdamped (relaxational) dynamics of an order-parameter field to the minimum of the potential in Eq. (4),i.e. the system is damped towards the equllibrium configuration. The resulting evolution equation is a first order time derivative for the order parameter field. We have studied the ordering dynamics of such a TDGL model in Ref. [9]. 
However, a microscopic derivation of the kinetic equation in a relativistic field theory using, e.g., the closed-time-path Green's function (CTPGF) formalism results in a second-order stochastic equation. Such a derivation has been done for scalar field theories $[17,18,19]$. A second-order TDGL equation has also been derived for the NJL model by Fu et al. [20] using the CTPGF method. More recently, a Langevin equation with an inertial term has been derived for the chiral order parameter field in a sigma model by Nahrgang et al. using an influence-functional method [21]. This model has been used to discuss the relaxational dynamics of the order parameter near the critical point $[22,23]$. Such a second order time derivative term, called an 'intertial term', is usually neglected in comparison to the damping term which is first order temporal derivative of the order parameter field. Given this background, it is relevant to investigate the effect of an inertial term on the ordering kinetics of the chiral transition. More generally, it is important to study the effect of an inertial term in domain growth problems. In spite of the intense interest in this area, this question has received almost no attention $[14,15]$. We will address this issue in the context of chiral transitions in the present work [10].

Thus, we consider a system whose evolution is described by the TDGL equation with an inertial term:

$$
\frac{\partial^{2}}{\partial t^{2}} M(\vec{r}, t)+\bar{\gamma} \frac{\partial M}{\partial t}=-\frac{\delta \Omega[M]}{\delta M(\vec{r}, t)}+\theta(\vec{r}, t),
$$

where $\bar{\gamma}$ is the dissipation coefficient. Here, $\theta(\vec{r}, t)$ is the noise term satisfying the fluctuationdissipation relation $\left(k_{B}=1\right)$ :

$$
\begin{aligned}
\langle\theta(\vec{r}, t)\rangle & =0, \\
\left\langle\theta\left(\vec{r}^{\prime}, t^{\prime}\right) \theta\left(\overrightarrow{r^{\prime \prime}}, t^{\prime \prime}\right)\right\rangle & =2 \bar{\gamma} T \delta\left(\overrightarrow{r^{\prime}}-\overrightarrow{r^{\prime \prime}}\right) \delta\left(t^{\prime}-t^{\prime \prime}\right) .
\end{aligned}
$$

We use the natural scales of order parameter, space and time to introduce dimensionless variables:

$$
\begin{aligned}
M & =M_{0} M^{\prime}, \quad M_{0}=\sqrt{|a| /|b|}, \\
\vec{r} & =\xi \vec{r}^{\prime}, \quad \xi=\sqrt{K /|a|}, \\
t & =\tau t^{\prime}, \quad \tau=1 / \sqrt{|a|} \\
\theta & =\left(|a|^{3 / 2} /|b|^{1 / 2}\right) \theta^{\prime} .
\end{aligned}
$$

Dropping the primes, we obtain the dimensionless TDGL equation:

$$
\frac{\partial^{2} M}{\partial t^{2}}+\gamma \frac{\partial M}{\partial t}=-\operatorname{sgn}(a) M-\operatorname{sgn}(b) M^{3}-\lambda M^{5}+\nabla^{2} M+\theta(\vec{r}, t),
$$

where $\gamma=\bar{\gamma} / \sqrt{|a|}, \operatorname{sgn}(x)=x /|x|$, and $\lambda=|a| d /|b|^{2}>0$. The dimensionless noise satisfies

$$
\begin{aligned}
\left\langle\theta\left(\overrightarrow{r^{\prime}}, t^{\prime}\right) \theta\left(\overrightarrow{r^{\prime \prime}}, t^{\prime \prime}\right)\right\rangle & =2 \varepsilon \delta\left(\overrightarrow{r^{\prime}}-\overrightarrow{r^{\prime \prime}}\right) \delta\left(t^{\prime}-t^{\prime \prime}\right), \\
\varepsilon & =\frac{\gamma T|b|}{|a|^{(5-d) / 2} K^{d / 2}},
\end{aligned}
$$

where $d$ is the spatial dimensionality.

Our results in this letter are presented in dimensionless units of space and time. To obtain these in physical units, one has to multiply by the appropriate dimensional quantities $\xi$ and $\tau$. 
For this, we need to estimate the strength of the interfacial energy $K$. The surface tension can be calculated as $\sigma=\sqrt{K}\left(|a|^{3 / 2} /|b|\right) \int d z\left(d M_{s} / d z\right)^{2}$, where $M_{s}(z)$ is the static kink solution of Eq. (8) with $\theta=0$. For quark matter, $\sigma$ is poorly known and varies from $10-100 \mathrm{MeV} / \mathrm{fm}^{2}$ at small temperatures [24]. On the other hand, recent estimates using effective models [25] like the NJL model and the Polyakov loop-quark-meson model suggest a lower value for surface tension: $\sigma \simeq 5-20 \mathrm{MeV}$. We take $\sigma \simeq 10 \mathrm{MeV} / \mathrm{fm}^{2}$.. For $T=10 \mathrm{MeV}$ and $\mu=321.75 \mathrm{MeV}$, we then estimate $\xi=\sqrt{K /|a|} \simeq 0.56 \mathrm{fm}$ and $\tau=1 / \sqrt{|a|} \simeq 5.1 \mathrm{fm}$ [9]. Let us first study the early time behavior of the deterministic version of Eq. (8) $(\theta=0)$. We linearize it around an extremum point $\bar{M}$ by replacing $M(\vec{r}, t)=\bar{M}+\phi(\vec{r}, t)$. In Fourier space, the linearized equation becomes

$$
\frac{\partial^{2}}{\partial t^{2}} \phi(\vec{k}, t)+\gamma \frac{\partial}{\partial t} \phi(\vec{k}, t)+\left(-\alpha+k^{2}\right) \phi(\vec{k}, t)=0
$$

where $\alpha=-f^{\prime \prime}(\bar{M})$. We have $\alpha>0$ when $\bar{M}$ is a local maximum, and $\alpha<0$ when $\bar{M}$ is a local minimum. Equation (10) is a homogeneous second-order differential equation, and one can write the general solution as

$$
\begin{aligned}
& \phi(\vec{k}, t)=A_{1} e^{\Lambda_{+}(\vec{k}) t}+A_{2} e^{\Lambda_{-}(\vec{k}) t} \\
& \Lambda_{ \pm}(\vec{k})=\frac{-\gamma \pm \sqrt{\gamma^{2}+4\left(\alpha-k^{2}\right)}}{2}
\end{aligned}
$$

Here $A_{1}$ and $A_{2}$ are constants. In the absence of dissipation $(\gamma=0)$, we have

$$
\Lambda_{ \pm}= \pm \sqrt{\alpha-k^{2}}
$$

First, consider the case $\alpha>0$. There is an instability for short wavelengths $(k<\sqrt{\alpha})$ with $\Lambda_{+}(\vec{k})>0$. Thus, there is an exponential growth of fluctuations about a local maximum of the free energy. This is valid even in the limit of no dissipation. For $\alpha<0$, there is no instability and fluctuations are exponentially damped. The damping is relaxational for $k^{2}<\left(\gamma^{2}-4|\alpha|\right) / 4$, and oscillatory for $k^{2}>\left(\gamma^{2}-4|\alpha|\right) / 4$. In the limit of no dissipation, the dynamics is purely oscillatory.

We study the phase transition kinetics for two different quench possibilities. First, we consider deep quenches through II $(b>0)$ from $a>0$ (with $M=0)$ to $a<0$, where the free energy has a double-well structure. Let us note here that we are quenching far below the line of second order transitions. The chirally-symmetric phase is now unstable, and evolves to the stable massive phase via spinodal decomposition. In our simulations of this case, we have used Eq. (8) with $a<0, b>0, \lambda=0.14$, corresponding to $(\mu, T)=(231.6 \mathrm{MeV}, 85 \mathrm{MeV})$ [9]. The appropriate form of the evolution equation is

$$
\frac{\partial^{2} M}{\partial t^{2}}+\gamma \frac{\partial M}{\partial t}=M-M^{3}-\lambda M^{5}+\nabla^{2} M+\theta(\vec{r}, t)
$$

We solve Eq. (13) numerically using a simple Euler-discretization scheme with initial velocity $\partial M /\left.\partial t\right|_{t=0}=0$. The initial state of the system is prepared as $M(\vec{r}, 0)=0 \pm \delta M(\vec{r}, 0)$, where $\delta M$ is uniformly distributed in $[-0.25,+0.25]$. This mimics the physical situation where small amplitude fluctuations are always present. Even if we start with a uniform initial state, thermal noise rapidly generates random fluctuations. 
Our numerical simulations are performed on a $d=3$ lattice of size $N^{3}(N=256)$, with periodic boundary conditions in all directions. The discretization mesh sizes are $\Delta x=1.0$ and $\Delta t=0.1$, obtained from the linear stability analysis of Eq. (13) [26,27]. We require that the Euler scheme must respect the stability properties of the homogeneous solutions of Eq. (13). The thermal noise $\theta(\vec{r}, t)$ is mimicked by uniformly-distributed random numbers between $\left[-A_{n}, A_{n}\right]$. In studies of phase-transition kinetics, it is known that statistical results are unchanged whether we use Gaussian noise or uniformly-distributed noise [26, 32]. The appropriate noise amplitude in our simulation is [28]

$$
A_{n}=\sqrt{\frac{3 \varepsilon}{(\Delta x)^{d} \Delta t}} .
$$

The results reported here correspond to $\varepsilon=0.008$, i.e., $A_{n}=0.5$. All statistical quantities are obtained as averages over 10 independent runs.

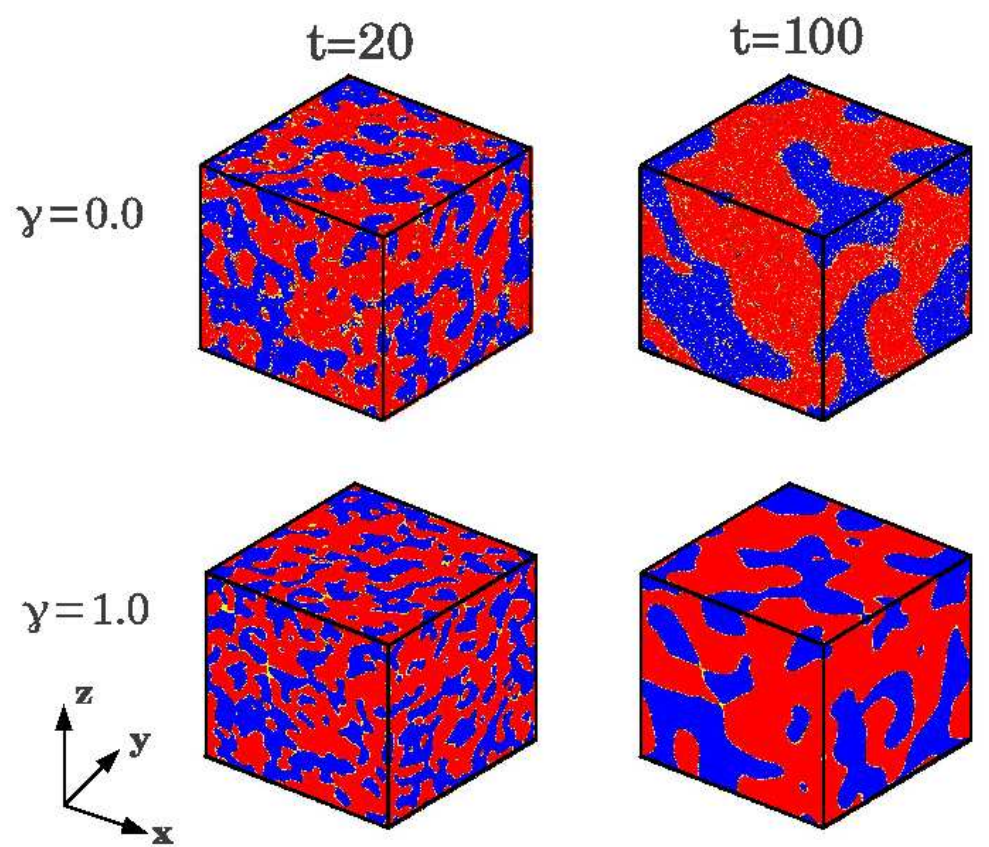

Figure 2: Domain growth for $\gamma=0.0,1.0$ after a quench through the second-order line (II) in Fig. 1. The snapshots show regions with $M \simeq+M_{+}$(marked red), $M \simeq 0$ (marked yellow), and $M \simeq-M_{+}$(marked blue) at $t=20,100$.

In Fig. 2, we show the ordering dynamics of Eq. (13) from a disordered initial state. To study the effect of inertia, we chose $\gamma=0.0$ (upper frames) and 1.0 (lower frames). The system rapidly evolves into domains of the massive phase with $M \simeq M_{+}$(marked red) and $M \simeq-M_{+}$(marked blue). The snapshots show the evolution at $t=20,100$. For $\gamma=0$, the dissipative term is absent, and we observed a rapid growth of domains (see the pattern at $t=20$ ). After the initial rapid growth, domain walls get fuzzier, and domains become less distinctive due to the oscillatory behavior of the system. We have also studied the time-dependence of the order-parameter value at a few spatial points in the $\gamma=0$ case. We observe the occurrence of flips from $\pm M_{+} \rightarrow \mp M_{+}$on extended 
time-scales. In spite of these, the domain morphology continues to coarsen as these oscillations are cooperative. For $\gamma=1$, the dissipative term is dominant and the ordering dynamics is analogous to that for the overdamped case [9].

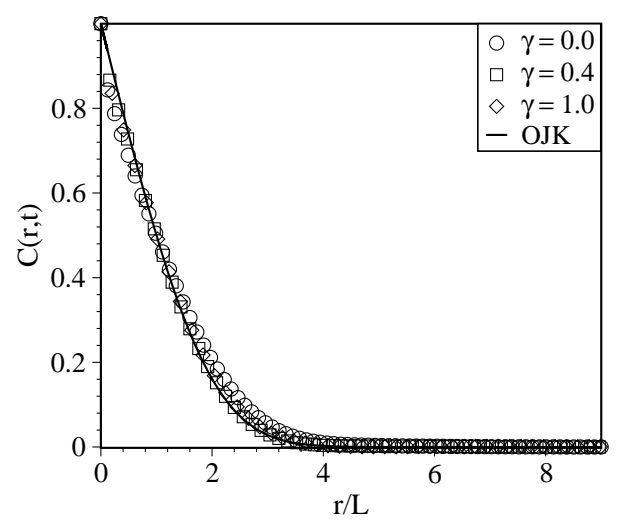

Figure 3: Plot of the scaled correlation function, $C(r, t)$ vs. $r / L$, for $\gamma=0,0.4,1.0$ at $t=20$. The length scale $L(t)$ isa defined as the distance over which the correlation function decays to half its maximum value $[C(r, t)=1$ at $r=0]$. The solid line denotes the OJK function in Eq. (17).

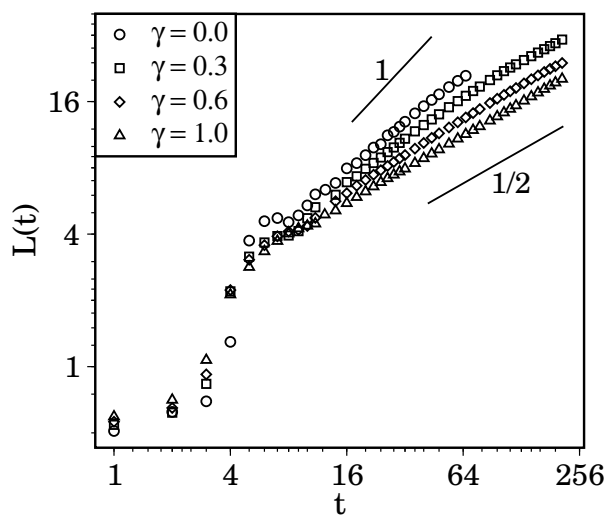

Figure 4: Time-dependence of domain size, $L(t)$ vs. $t$, for the evolution depicted in Fig. 2. There is a crossover at $t_{c} \sim \gamma^{-1}$ from an early-time inertial growth $\left[L(t) \sim t(\ln t)^{1 / 2}\right]$ to a late-time Cahn-Allen (CA) growth $\left[L(t) \sim t^{1 / 2}\right]$.

The system is characterized by a single length scale $L(t)$ as the pattern morphology does not change in time apart from a scale factor. The morphology is quantitatively studied using the correlation function [15]:

$$
C(\vec{r}, t)=\frac{1}{V} \int d \vec{R}[\langle M(\vec{R}, t) M(\vec{R}+\vec{r}, t)\rangle-\langle M(\vec{R}, t)\rangle\langle M(\vec{R}+\vec{r}, t)\rangle] .
$$

Here, $V$ denotes the volume of the system, and the angular brackets denote an average over independent runs. The evolution morphologies are isotropic, so we compute the spherically-averaged correlation function $C(r, t)$ with $r=|\vec{r}|$. The existence of the characteristic scale results in a $d y$ namical scaling of the correlation function:

$$
C(r, t)=g[r / L(t)]
$$


We have confirmed numerically (not shown here) that the correlation functions at different times obey dynamical scaling for different $\gamma$-values. In Fig. 3, we plot the scaled correlation function, $C(r, t)$ vs. $r / L$, for $\gamma=0,0.4,1.0$ at $t=20$. The length scale $L(t)$ is defined as the distance over which the correlation function decays to half its maximum value $[C(r, t)=1$ at $r=0]$. Notice that the scaling functions are numerically indistinguishable showing that the evolution morphologies are the same for different values of $\gamma$. The solid line denotes the Ohta-Jasnow-Kawasaki (OJK) function $[29,30]$ :

$$
g_{\mathrm{OJK}}(x)=\frac{2}{\pi} \sin ^{-1}\left(e^{-x^{2} / 2}\right),
$$

which characterizes ordering dynamics for the $M^{4}$-potential in the overdamped limit, i.e., without inertial terms. Clearly, our numerical data is well-described by the OJK function.

In Fig. 4, we plot $L(t)$ vs. $t$ on a $\log -\log$ scale for several values of $\gamma$. As usual, $L(t)$ shows a power-law behavior $\left[L(t) \sim t^{\phi}\right]$, but there is a distinct crossover in the exponent $\phi$ as $\gamma$ is varied.

This can be understood by considering the deterministic version $(\theta=0)$ of Eq. (13), which leads to the domain growth equation as[10]

$$
\frac{d^{2} L}{d t^{2}}+\gamma \frac{d L}{d t}=\frac{\sigma}{L}
$$

where $\sigma / L$ is identified as the curvature for a domain of size $L$. At short times $\left(t \ll t_{c}\right)$, the growth law is fixed by the inertial term as [31]

$$
L(t) \sim \sqrt{\sigma} t[\ln (\sqrt{\sigma} t)]^{1 / 2} .
$$

The long-time $\left(t \gg t_{c}\right)$ kinetics is determined by the dissipative term as

$$
L(t) \sim\left(\frac{\sigma t}{\gamma}\right)^{1 / 2}
$$

which is the usual Cahn-Allen (CA) growth law [15]. The crossover time scales as $t_{c} \sim \gamma^{-1}$. In Fig. 4, we have plotted straight lines corresponding to $L(t) \sim t$ and $L(t) \sim t^{1 / 2}$, the two limiting behaviors of the growth law.

Next, we consider shallow quenches through I to the point marked by a asterisk in Fig. 1. This case is studied using Eq. (8) with $a>0, b<0, \lambda=0.14$, which is equivalent to $(\mu, T)=(321.75$ $\mathrm{MeV}, 10 \mathrm{MeV}$ ) [9]. The corresponding kinetic equation is

$$
\frac{\partial^{2} M}{\partial t^{2}}+\gamma \frac{\partial M}{\partial t}=-M+M^{3}-\lambda M^{5}+\nabla^{2} M+\theta(\vec{r}, t) .
$$

The initial state with massless quarks $(M=0)$ is now a metastable state of the potential, and phase separation proceeds via nucleation and growth of droplets of $M= \pm M_{+}$. Therefore, the thermal noise $\theta(\vec{r}, t)$ must be sufficiently large to enable the system to escape from the metastable state on a reasonable time-scale: a suitable value for $\lambda=0.14$ is $\varepsilon=0.6$. However, the asymptotic behavior of domain growth in both the unstable and metastable cases is insensitive to the noise term [32]. 

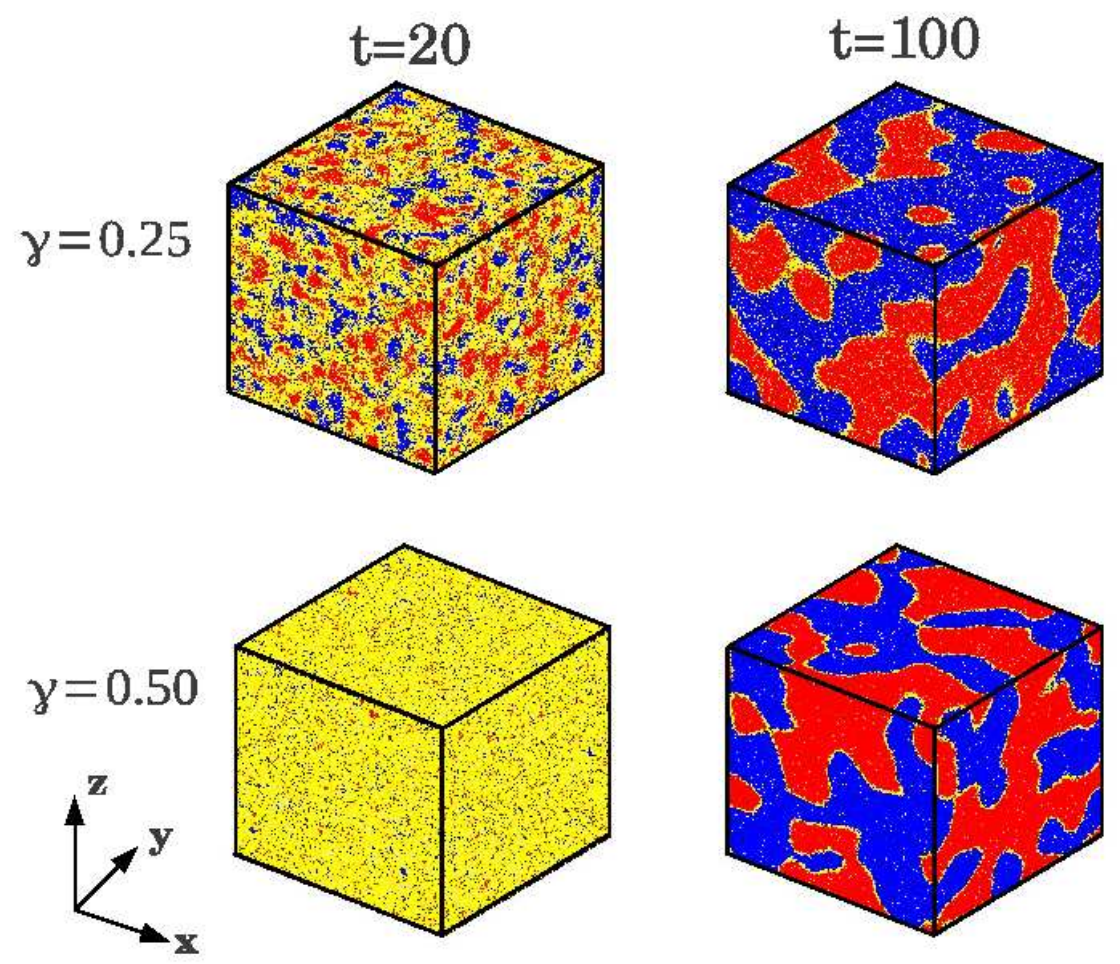

Figure 5: Analogous to Fig. 2 but for a shallow quench through the first-order line (I) in Fig. 1 . Notice that the metastable patches $(M \simeq 0$, marked yellow) at $t=20$ are absent at later times.

In Fig. 5, we show the ordering kinetics of Eq. (21) for $\gamma=0.25,0.5$. Typically, the evolution of the system begins with the nucleation of droplets in the early stages: droplets larger than a critical size $R_{c}$ (supercritical) grow, whereas those with $R<R_{c}$ (subcritical) shrink. In the present simulation, the critical radius of the bubble $R_{c} \simeq 8$ dimensionless units. If we convert this into physical units, $R_{c} \simeq 4.5 \mathrm{fm}$.

The droplets grow very rapidly and fuse to form bi-continuous domain structures, a characteristic of late-stage domain growth. The effect of dissipation on nucleation and growth can be understood by comparing the evolution patterns at different $\gamma$-values. The system takes more time to nucleate for extreme $\gamma$-values (i.e., $\gamma \rightarrow 0$ and $\gamma \rightarrow \infty$ ). To understand this behavior, we follow Hanggi's discussion [33] of Kramer's escape problem for a barrier. Hanggi studies the crossover time from $M=0$ (the metastable state) to $M=M_{+}$(the stable state) in the homogeneous version of Eq. (21). This crossover time is proportional to the nucleation time $t_{n}$ in our domain growth problem. We designate $\omega_{b}$ as the natural vibration frequency about the barrier location $\left(M_{-}\right)$. For moderate to large dissipation $\left(\gamma \gg \omega_{b}\right)$, the nucleation time

$$
t_{n} \sim\left(\sqrt{\frac{\gamma^{2}}{4}+\omega_{b}^{2}}-\frac{\gamma}{2}\right)^{-1}
$$


so that $t_{n} \sim \gamma$ as $\gamma \rightarrow \infty$. For small dissipation $\left(\gamma \ll \omega_{b}\right)$, we have

$$
t_{n} \sim \frac{1}{\gamma}
$$

so that $t_{n} \rightarrow \infty$ as $\gamma \rightarrow 0$.

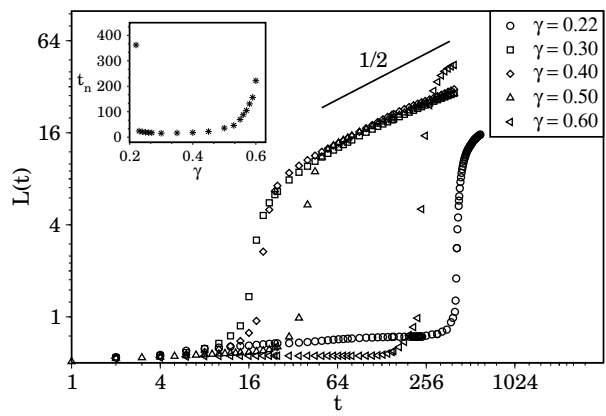

Figure 6: Time-dependence of the domain size, $L(t)$ vs. $t$, for nucleation and growth with different $\gamma$ values. There is no growth in the early stages when droplets are being nucleated. The asymptotic growth is consistent with the CA growth law, $L(t) \sim t^{1 / 2}$. The inset shows the $\gamma$-dependence of the nucleation time $t_{n}$ for the onset of domain growth.

In Fig. 6, we plot the domain size $[L(t)$ vs. $t]$ on a log-log scale: the growth process begins once the nucleation of droplets is over. The onset time for domain growth is the nucleation time $t_{n}$, which is shown in the inset of Fig. 6 for different values of $\gamma$. Notice that $t_{n} \rightarrow \infty$ as $\gamma \rightarrow 0$ or $\gamma \rightarrow \infty$, as expected. The intermediate and asymptotic growth regimes are similar to those described for spinodal decomposition, i.e., a crossover from $L(t) \sim t(\ln t)^{1 / 2}$ to $L(t) \sim t^{1 / 2}$. In Fig. 6, we have focused on the $\gamma$-dependence of $t_{n}$, rather than the asymptotic growth laws.

In summary, we have studied the kinetics of chiral phase transitions in QCD subsequent to sudden changes in system parameters. To understand the kinetics, we must first obtain the phase diagram. In terms of the quark degrees of freedom, the phase diagram is obtained in the $(\mu, T)$ plane using the Nambu-Jona-Lasinio (NJL) model [9]. An equivalent coarse-grained description is obtained from an $M^{6}$-Landau free energy.

The chiral kinetics is modeled via the nonlinear TDGL equation with dissipation and noise, and we consider both the overdamped [9] and inertial cases [10]. We study quenches through the first-order (I) or second-order (II) transition lines in Fig. 1. For quenches through II and deep quenches through I, the massless phase is spontaneously unstable and evolves to the massive phase via spinodal decomposition. For shallow quenches through I, the massless phase is metastable and the chiral transition proceeds via the nucleation and growth of droplets of the massive phase. The merger of these droplets results in late-stage domain growth similar to that for the unstable case. In all cases, the asymptotic growth process exhibits dynamical scaling, and the growth law is $L(t) \sim t^{1 / 2}$. The inertial term gives a pre-asymptotic regime of faster growth with $L(t) \sim t(\ln t)^{1 / 2}$, and the crossover time to $t^{1 / 2}$-growth scales as $t_{c} \sim \gamma^{-1}$, where $\gamma$ is the dissipation constant.

In the context of heavy-ion collisions, given the uncertain values of dimensional quantities for quark matter (e.g., surface tension, dissipation), it is not clear whether the system equilibrates completely within the life-time of the fireball. If the system is almost equilibrated, the features of the coarsening morphology are similar for quenches through both first- and second-order lines in the 
phase diagram. However, if the equilibration time-scale is much larger than the fireball life-time, the morphology is very different for quenches through the first-order line, with the system evolving through nucleation of droplets. The signatures of such a quench through first-order transition are experimentally relevant because they imply the existence of a critical end point (CEP) in the QCD phase diagram. As a matter of fact, experimental studies of such signatures may be more convenient than directly searching for the CEP via critical fluctuations. To date, the latter approach has not provided conclusive evidence of the existence of a CEP, presumably due to the smallness of the critical region.

\section{References}

[1] M. Alford, K. Rajagopal and F. Wilczek, Phys. Lett. B 422, 247 (1998); R. Rapp, T. Schafer, E.V. Shuryak and M. Velkovsky, Ann. Phys. 280, 35 (2000); O. Scavenius et al., Phys. Rev. C 64, 045202 (2001).

[2] Z. Fodor and S. Katz, JHEP 0203, 014 (2002); P. de Forcrand and O. Phillipsen, Nucl. Phys. B 642, 290 (2002); C.R. Alton et al., Phys. Rev. D 66, 074507 (2002); Phys. Rev. D 68, 014507 (2003).

[3] M.A. Stephanov, Int. J. Mod. Phys. A 20, 4387 (2005).

[4] B. Mohanty, Nucl. Phys. A 830, 899 (2009); T. Schuster, Proc. Sci. CPOD2009, 029 (2009); G. Stefanek, Proc. Sci. CPOD2009, 049 (2009).

[5] E.S. Fraga and G. Krein, Phys. Lett. B 614, 181 (2005).

[6] A. Bessa, E.S. Fraga and B.W. Mintz, Phys. Rev. D 79, 034012 (2009).

[7] V.V. Skokov and D. N. Voskresensky, Nucl. Phys. A 828401 (2009).

[8] J. Randrup, Phys. Rev. C 79, 054911 (2009); Phys. Rev. C 82, 034902 (2010).

[9] A. Singh, S. Puri and H. Mishra, Nucl. Phys. A 864, 176 (2011).

[10] A. Singh, S. Puri and H. Mishra, Nucl. Phys. A 908, 18 (2013),ibid Euro. Phys. Lett. (to appear).

[11] S.P. Klevansky, Rev. Mod. Phys. 64, 649 (1992).

[12] C. Sasaki, B. Friman and K. Redlich, Phys. Rev. D 77, 034024 (2008); M. Iwasaki, Phys. Rev. D 70, 114031 (2004); H. Fujii and M. Ohtani, Phys. Rev. D 70, 014016 (2004).

[13] H. Mishra and S.P. Misra, Phys. Rev. D 48, 5376 (1993).

[14] A.J. Bray, Adv. Phys. 43, 357 (1994).

[15] S. Puri and V.K. Wadhawan (eds.), Kinetics of Phase Transitions, CRC Press, Boca Raton, Florida (2009).

[16] A. Berera, M. Gleiser and R. Ramos, Phys. Rev. D D58(1998) 123508; M. bastero-Gil, A. Berera, R. Ramos , JCAP 1109(2011)033; M. bastero-Gil, A. Berera, R. Ramos , J. Rosa, JCAP 1301(2013)016.

[17] M. Gleiser and R.O. Ramos, Phys. Rev. D 50, 2441 (1994).

[18] D.H. Rischke, Phys. Rev. C 58, 2331 (1998).

[19] D. Boyanovsky, H.J. de Vega, R. Holman and J. Salgado, Phys. Rev. D 59, 125009 (1999).

[20] W. Fu, D. Huang and F. Wang, Nucl. Phys. A 849, 203 (2011).

[21] M. Nahrgang, S. Leupold, C. Herold and M. Bleicher, Phys. Rev. C 84, 024912 (2011). 
[22] M. Nahrgang, S. Leupold and M. Bleicher, Phys. Lett. B711,109, (2012).

[23] M. Nahrgang, M. Bleicher, S. Leupold and I. Mishustin, J Phys. G.40 055108, (2013); C. Herold, M. Nahrgang, I. Mishustin and M. Bleicher, Phys Rev C87, 014907, (2013).

[24] H. Heiselberg, C.J. Pethick and E.F. Staubo, Phys. Rev. Lett. 70, 1355 (1993).

[25] L. F. Palhares and E. S. Fraga, Phys. Rev. D 82, 125018 (2010); M. B. Pinto, V. Koch and J. Randrup, Phys. Rev. C 86, 025203 (2012).

[26] Y. Oono and S. Puri, Phys. Rev. Lett. 58, 836 (1987); Phys. Rev. A 38, 434 (1988); S. Puri and Y. Oono, Phys. Rev. A 38, 1542 (1988).

[27] T.M. Rogers, K.R. Elder and R.C. Desai, Phys. Rev. B 37, 9638 (1988).

[28] S. Puri and K. Binder, Phys. Rev. Lett. 86, 1797 (2001); Phys. Rev. E 66, 061602 (2002).

[29] T. Ohta, D. Jasnow and K. Kawasaki, Phys. Rev. Lett. 49, 1223 (1982); Y. Oono and S. Puri, Mod. Phys. Lett. B 2, 861 (1988).

[30] A.J. Bray and S. Puri, Phys. Rev. Lett. 67, 2670 (1991).

[31] C.M. Bender and S.A. Orszag, Advanced Mathematical Methods for Scientists and Engineers, McGraw-Hill, Singapore (1984).

[32] S. Puri and Y. Oono, J. Phys. A 21, L755 (1988).

[33] P. Hanggi, J. Stat. Phys. 42, 105 (1986). 